\title{
A Stable, Wideband Tunable, Near Transform-Limited, Graphene- Mode-Locked, Ultrafast Laser
}

\author{
Zhipei Sun ${ }^{1}$, Daniel Popa ${ }^{1}$, Tawfique Hasan ${ }^{1}$, Felice Torrisi ${ }^{1}$, Fengqiu Wang ${ }^{1}$, Edmund J. R. Kelleher ${ }^{2}$, John C. \\ Travers $^{2}$, Valeria Nicolosi ${ }^{3}$, and Andrea C. Ferrari ${ }^{1}(\bowtie)$ \\ ${ }^{1}$ Department of Engineering, University of Cambridge, Cambridge CB3 0FA, UK \\ ${ }^{2}$ Physics Department, Imperial College, London SW7 2AZ, UK \\ ${ }^{3}$ Department of Materials, University of Oxford, Oxford OX1 3PH, UK \\ Received: 9 July 2010 / Accepted: 3 August 2010 \\ C The Author(s) 2010. This article is published with open access at Springerlink.com
}

\begin{abstract}
We report an ultrafast laser mode-locked with a graphene saturable absorber. The linear dispersions of the Dirac electrons in graphene enable wideband tunability. We get 1 ps pulses, tunable between 1525 and $1559 \mathrm{~nm}$, with stable mode-locking, insensitive to environmental perturbations.
\end{abstract}

\section{KEYWORDS}

Graphene, saturable absorber, mode-locking, fiber laser

\section{Introduction}

Ultrafast passively mode-locked fiber lasers with spectral tuning capability have widespread applications in biomedical research, spectroscopy, and telecommunications [1-3], due to their simplicity, compactness and efficient heat dissipation [2-6]. Currently, the dominant technology is based on semiconductor saturable absorber mirrors (SESAMs) [2-4]. However, these have a narrow tuning range, and require complex fabrication and packaging [2,7]. A simple, costeffective alternative is to use carbon nanotubes (CNTs) [8-36]. In CNTs the diameter controls the bandgap, thus, defining the operating wavelength. Broadband tunability is possible using CNTs with a wide diameter distribution [14, 36, 37]. However, when operating at a particular wavelength, CNTs not in resonance are not used and give insertion losses.

After the first demonstration of a graphene-based

$\overline{\text { Address correspondence to acf26@ }}$ cam.ac.uk mode-locker [32], a variety of lasers were reported exploiting graphene saturable absorbers for ultrafast pulse generation at 1 and $1.5 \mu \mathrm{m}$ [38-46]. Reference [38] explained the fundamentals of the photo-excited carrier dynamics, which leads to Pauli-blocking and thus, saturable absorption, with good agreement between theory and experiment. The linear dispersion of the Dirac electrons in graphene provides an ideal solution for wideband ultrafast pulse generation [38]. Wavelength tuning in graphene-based lasers has recently been achieved by exploiting fiber birefringence [44, 45]. However, fiber birefringence is sensitive to temperature fluctuations and other environmental instabilities [47], making this approach not ideal for long-term stability, a key requirement for mode-locked lasers used in practical applications. The 49 ps pulse width reported in Ref. [45] can be easily achieved by Q-switching [48], thus does not demonstrate the merits of the mode-locking technology. Furthermore, 
the output pulses in Ref. [45] are strongly chirped, with a time-bandwidth product (TBP) of 44.3. Therefore these are not transform-limited, i.e., the duration is not the shortest allowed for a given pulse spectral width. For a range of applications, transform-limited pulses are a prerequisite [49]. For example, in optical fiber communications, transform-limited pulses minimize the effect of chromatic dispersion during propagation in the fiber, thus, maximizing the transmission distance [49].

Here, we demonstrate an ultrafast tunable fiber laser mode-locked by a graphene-based saturable absorber, with stable mode-locking over a range of $34 \mathrm{~nm}$, insensitive to environmental perturbations. The tuning range is limited only by the tunable filter, with a wider tuning range potentially obtainable with a broader filter. The output pulse duration is $\sim 1$ ps. The TBP is 0.4 , and the pulses are near-transform-limited, and thus, viable for practical applications.

\section{Experimental}

\subsection{Device fabrication and characterization}

The saturable absorber is prepared by surfactantassisted liquid phase exfoliation of graphite flakes [38]. To obtain an aqueous dispersion, $1.2 \mathrm{wt} . \%$ of micronized graphite flakes are sonicated for $\sim 3 \mathrm{~h}$ in a bath sonicator with $0.5 \mathrm{wt}$.\% sodium deoxycholate (SDC), a bile salt surfactant. These surfactants, because of their flat molecular structure, adsorb readily on hydrophobic graphite surfaces $[50,51]$. Their $\beta$ side has a larger contact area $\left(1.8-3 \mathrm{~nm}^{2}\right)$ per surfactant molecule [52] than linear chain surfactants (e.g., sodium dodecylbenzene sulfonate (SDBS) $[53,54]$ ), which adsorb on graphitic surfaces through their alkyl chains [55]. The unexfoliated graphitic particles are allowed to settle for $10 \mathrm{~min}$ after sonication before being centrifuged at $10,000 \mathrm{r} / \mathrm{min}(17,000 \mathrm{~g})$ for $1 \mathrm{~h}$. The top $70 \%$ of the dispersion is decanted for characterization and composite fabrication.

A PerkinElmer spectrometer is used for absorption measurements. Raman measurements on dropcast flakes are carried out using a Renishaw spectrometer at $514.5 \mathrm{~nm}$ excitation. Transmission electron microscopy (TEM) images are recorded using a JEM-3000F field emission gun (FEG) TEM at $300 \mathrm{kV}$. For TEM, the dispersions were dropped onto a lacey carbon support grid (400 mesh).

The absorption spectrum of the centrifuged dispersion diluted to $10 \%$ is shown in Fig. 1(a). The spectrum is mostly featureless [38, 56, 57] a part from a peak in the UV region due to the exciton-shifted van Hove singularity in the graphene density of states [57, 58]. Absorption spectroscopy may be used to estimate the concentration of single-wall nanotubes (SWNTs) $[59,60]$ and graphene flakes $[53,61]$ from the BeerLambert law. Using the absorption coefficient of 1390 $\mathrm{L} \cdot \mathrm{g}^{-1} \cdot \mathrm{m}^{-1}$ for aqueous graphite dispersions at $660 \mathrm{~nm}$ as empirically determined in Ref. [53], we estimate a graphene concentration of $\sim 0.18 \mathrm{~g} \cdot \mathrm{L}^{-1}$ in the centrifuged dispersion.

Figure 1(b) shows a TEM image of a folded single layer graphene (SLG). TEM statistics indicates that in the dispersions were composed of $26 \%$ SLG, $22 \%$ bilayer (BLG), with the remainder being few-layer graphene (FLG) flakes. Of the BLGs, $40 \%$ are folded SLGs.

The Raman spectrum of a representative exfoliated graphene flake is shown Fig. 1(c). In addition to the $G$ and $2 D$ peaks, the spectrum shows significant $\mathrm{D}$ and $\mathrm{D}^{\prime}$ intensities. The $\mathrm{G}$ peak corresponds to the $E_{2 g}$ phonon at the Brillouin zone centre. The $D$ peak originates from the breathing modes of $\mathrm{sp}^{2}$ rings and requires a defect for its activation by double resonance (DR) [62-64]. The 2D peak is the second order of the D peak. It is a single band in SLG, but splits in four subbands in BLG, reflecting the evolution of the band structure [62]. The 2D peak is always seen, even in the absence of a D peak, as no defects are required for the activation of two phonons with the same momentum, one backscattering from the other. DR can also arise intra-valley, i.e., connecting two points belonging to the same cone around $\mathrm{K}$ or $\mathrm{K}^{\prime}$, giving the $\mathrm{D}^{\prime}$ peak. The large $\mathrm{D}$ intensity observed in Fig. 1(c) is not due to a large amount of disorder, otherwise the band would be much broader, and G would merge with $\mathrm{D}^{\prime}$ [63]. Instead, we assign it to the edges of the sub-micrometer flakes [65]. We note that 2D, although broader than in pristine graphene [62], is still a single Lorentzian. Thus, even if FLG are present, these behave as decoupled SLGs, retaining the linear dispersion of Dirac fermions [66].

To prepare the composite, aqueous solutions 


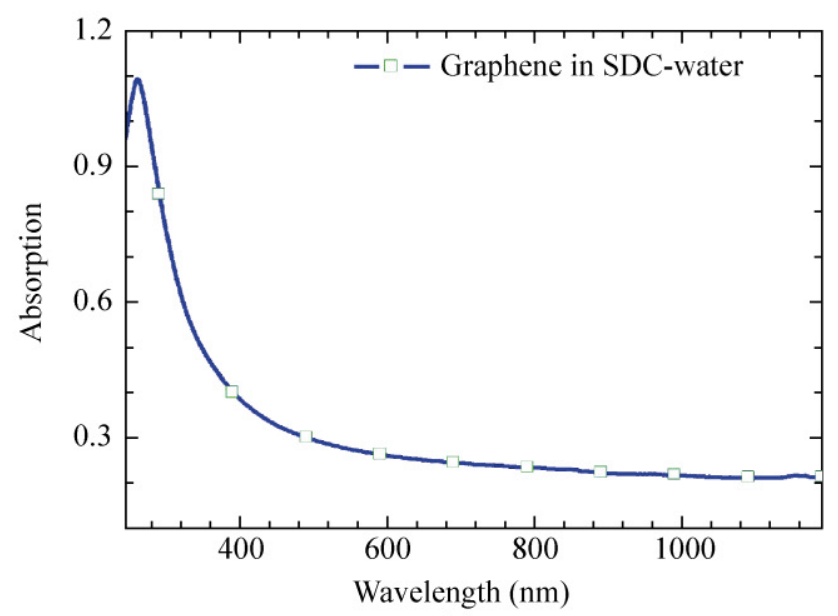

(a)

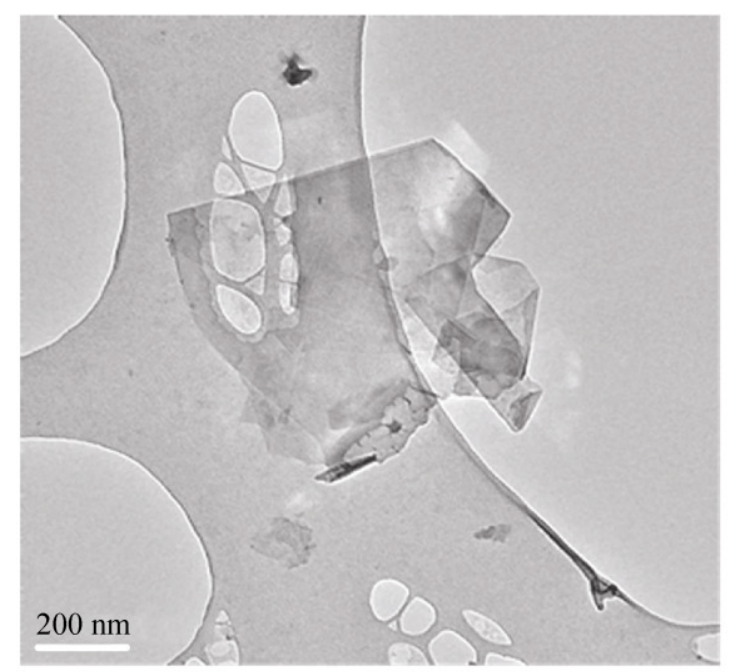

(b)

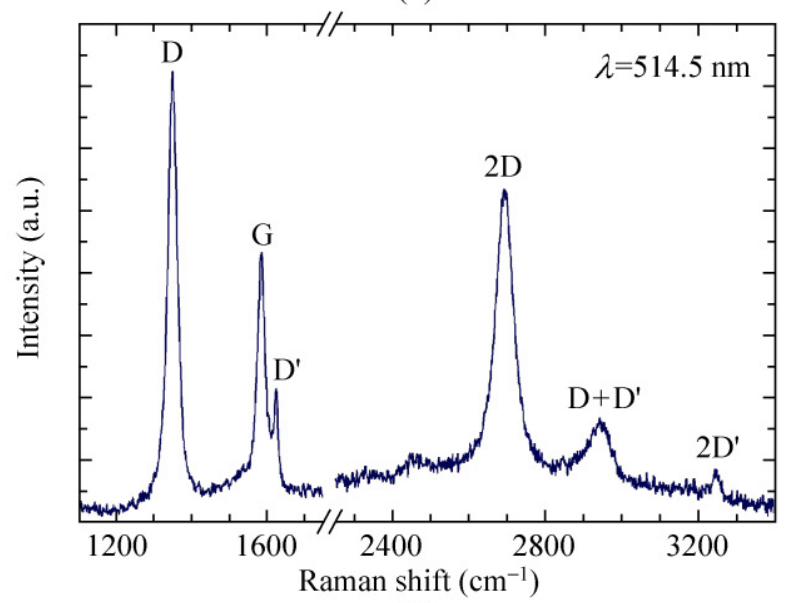

(c)

Figure 1 (a) Absorption spectrum of graphene dispersed in aqueous SDC solution. The absorption contributions from water and the surfactant have been subtracted. (b) TEM image of a folded flake. (c) Raman spectrum of a flake deposited on a $\mathrm{Si}$ wafer containing $120 \mathrm{mg}$ polyvinyl alcohol (PVA; Wako Chemicals) are sonicated with $4 \mathrm{~mL}$ of centrifuged graphene dispersion. The water is then slowly evaporated in a desiccator at room temperature, resulting in a $\sim 50 \mu \mathrm{m}$ thick graphene-PVA (GPVA) composite $[32,38]$. Compared to other fabrication strategies for graphene saturable absorbers [39-46], our approach is easily scalable, and allows integration into various photonic systems [32, 38, 46].

Power-dependent absorption at six wavelengths is measured using an all-fiber based setup described in Ref. [32] (see Fig. 2) by sandwiching the GPVA film between two fiber connectors (as shown in Fig. 3(a)). The absorption decreases by $\sim 4.5 \%$ due to saturation when the incident average power is increased to

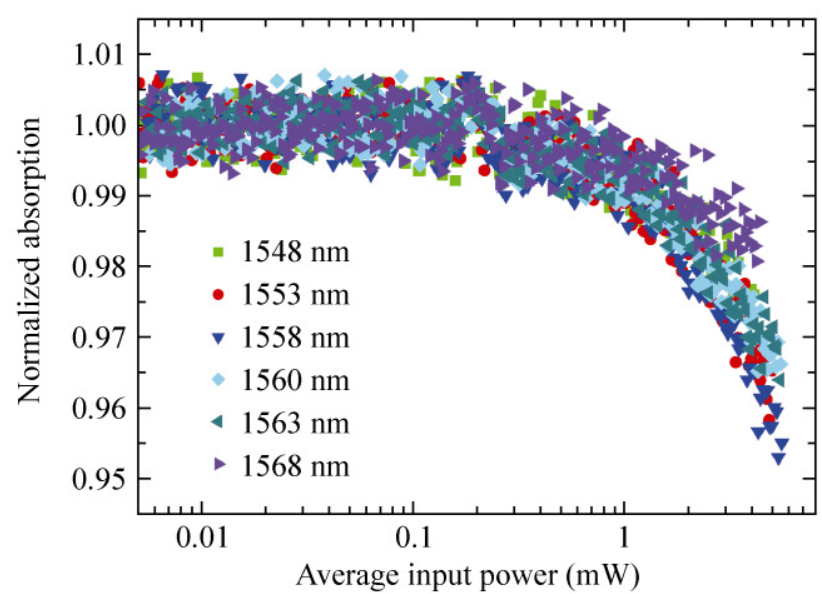

Figure 2 Power-dependent absorption at six wavelengths. Input repetition rate: $\sim 38 \mathrm{MHz}$; pulse duration: $\sim 580 \mathrm{fs}$

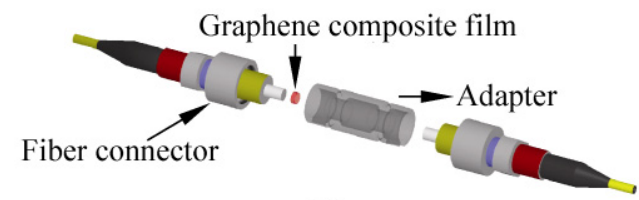

(a)

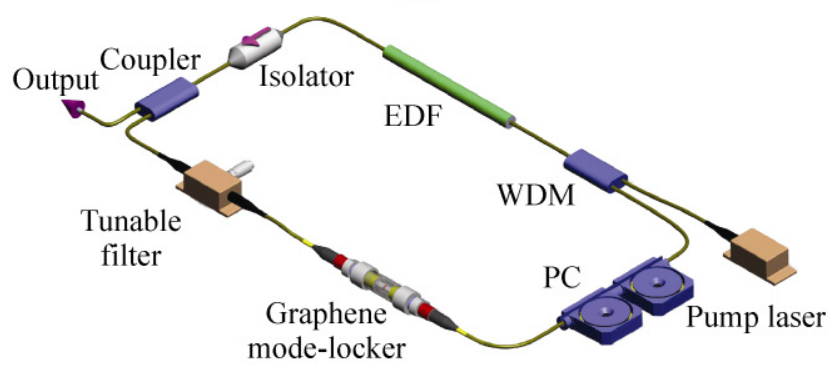

(b)

Figure 3 (a) Graphene saturable absorber assembly. (b) Tunable laser setup 
$5.35 \mathrm{~mW}\left(266 \mathrm{MW} / \mathrm{cm}^{2}\right.$ power density) at $1558 \mathrm{~nm}$, independent of wavelength. The nonlinear operation in terms of modulation depth and non-saturable absorption is comparable to that of the CNT-based devices reported in the Refs. [14, 19, 21, 23].

Note that the data in Fig. 2 are just limited by the availability of our pump wavelengths. Saturable absorption is expected over a much wider spectral range due to the linear dispersion of the graphene electrons $[32,38,46]$.

\subsection{Laser setup}

The packaged GPVA is then integrated to form a tunable ultrafast laser, as shown in Fig. 3(b). The cavity consists of an erbium-doped fiber (EDF), followed by an optical isolator (ISO), a fused fiber coupler, a tunable filter with a $12.8 \mathrm{~nm}$ bandwidth, a polarization controller (PC), and a fused fiber wavelength division multiplexer (WDM). A $1.2 \mathrm{~m}$ EDF (Fibercore) is backward pumped by a $976 \mathrm{~nm}$ laser diode (Bookham LC96V74-20R) through the WDM. Unidirectional operation is imposed by the ISO. The PC is used to adjust polarization for mode-locking optimization. The $20 \%$ port of the 20/80 coupler feeds the pulses back into the cavity. The total cavity length is $\sim 26.3 \mathrm{~m}$. The lasing wavelength is selected by the in-line tunable filter. The band-pass filter angle is controlled by a micrometer screw, providing continuous tunability from 1530 to $1555 \mathrm{~nm}$. This ensures stable mode-locking, independent of temperature and environmental fluctuations, unlike the wavelength selection strategy based on tuning fiber birefringence with a polarization controller used in Refs. [44, 45]. The pump and average output power are monitored by a photodiode, while the pulse duration and spectrum are recorded using a second harmonic generation (SHG) intensity autocorrelator (APE Pulsecheck 50) and an optical spectrum analyzer (Anritsu MS9710B), respectively.

\section{Results and discussion}

Continuous wave operation starts at a pump power of $\sim 6 \mathrm{~mW}$, giving an output power of $\sim 35 \mu \mathrm{W}$. Singlepulse mode-locking is observed at a pump power of $\sim 20 \mathrm{~mW}$. The output wavelength is tunable from 1525 to $1559 \mathrm{~nm}$ (see Fig. 4(a)); this is limited by the tunable filter used in our experiment, not by the graphene-saturable absorber. The full width at half maximum (FWHM) spectral bandwidth at a representative output wavelength $(1553 \mathrm{~nm})$ is $\sim 3 \mathrm{~nm}$. Typical soliton sidebands are observed, due to periodic intracavity perturbations [67]. The autocorrelation traces are shown in Fig. 4(b). Assuming a sech ${ }^{2}$ pulse shape, the deconvolved pulse duration is $\sim 1$ ps at $1553 \mathrm{~nm}$. Pulse durations and time-bandwidth products (TBP) at different output wavelengths are shown in Fig. 5. The TBP at $1553 \mathrm{~nm}$ is $\sim 0.4$. The deviation from 0.315, expected for transform-limited sech ${ }^{2}$ pulses, indicates minor chirping [47]. The nominal tuning range of our filter is $1530-1555 \mathrm{~nm}$, but it can also operate between

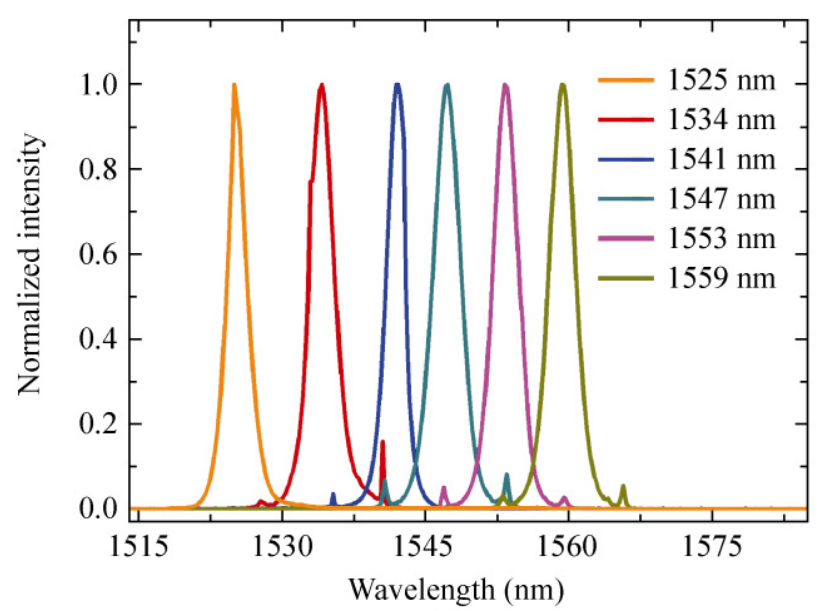

(a)

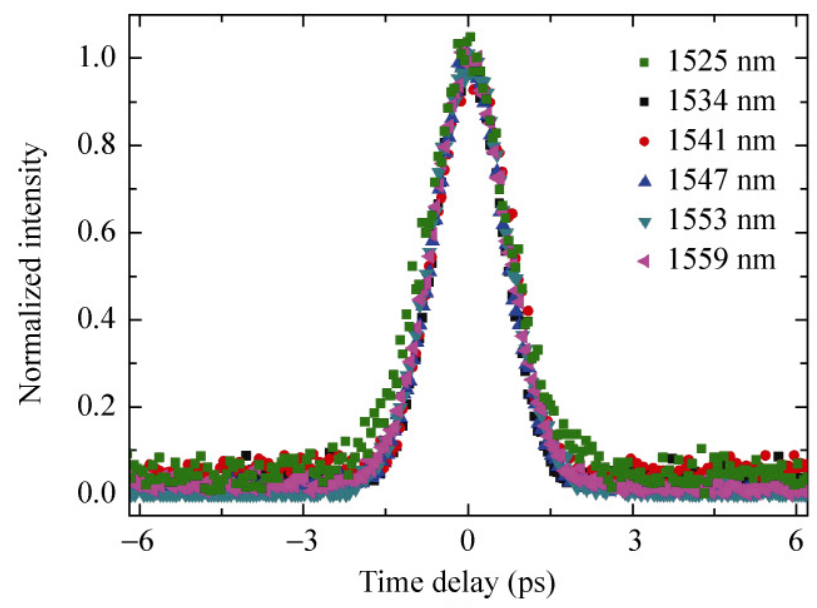

(b)

Figure 4 (a) Output spectra. (b) Autocorrelation traces at different wavelengths 


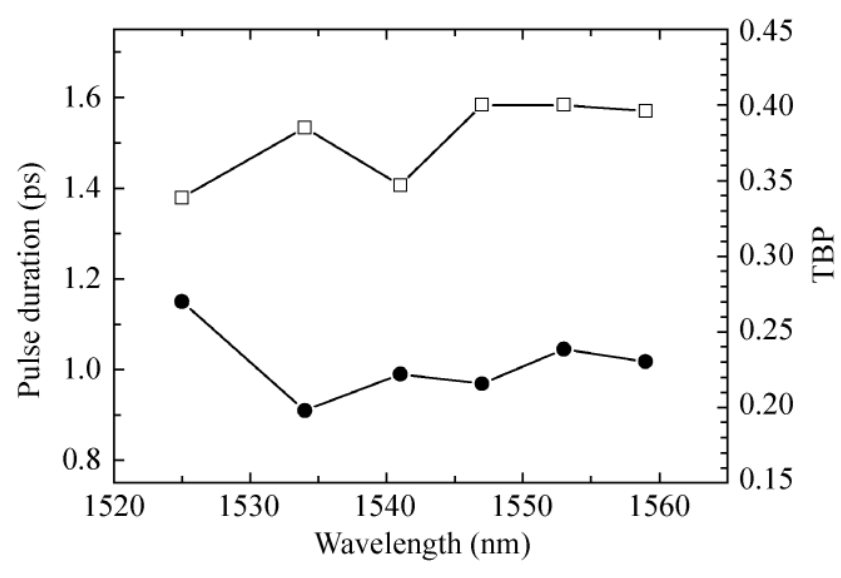

Figure 5 Output pulse duration $(\bullet)$ and time-bandwidth product $(\square)$ as a function of wavelength

1525 and $1530 \mathrm{~nm}$ and between 1555 and $1559 \mathrm{~nm}$, at the expense of increased insertion losses. Thus, the shortest and longest wavelengths that can be achieved are 1525 and $1559 \mathrm{~nm}$. The pulse at $1525 \mathrm{~nm}$ is slightly longer, $\sim 1.15 \mathrm{ps}$, possibly due to the higher losses. The average output power is $\sim 1 \mathrm{~mW}$, with $\sim 125 \mathrm{pJ}$ pulse energy.

The stability is characterized from radio-frequency (RF) measurements of the output intensity [68]. The repetition rate is $\sim 8 \mathrm{MHz}$, corresponding to $\sim 125.5 \mathrm{~ns}$ round-trip time, as shown in Fig. 6(a). Figure 6(b) shows the RF spectrum of the fundamental harmonic frequency. The peak to pedestal extinction is $\sim 80 \mathrm{~dB}$, indicating low amplitude noise fluctuations [68]. Compared to previous tunable, graphene modelocked lasers [44,45], we can achieve significantly lower fluctuations, and much shorter, near transformlimited pulse durations, thus, showcasing the wideband operation capability of graphene and its potential as a wideband mode-locker.

\section{Conclusion}

We have demonstrated a stable, wideband tunable ultrafast laser using a graphene-based saturable absorber, generating near transform-limited $\sim 1 \mathrm{ps}$ pulses. The tunable output spectral range is from 1525 to $1559 \mathrm{~nm}$. Such a wideband mode-locker is ideal for ultrafast light sources covering a large spectral range for applications such as metrology, spectroscopy, and biomedical diagnostics.

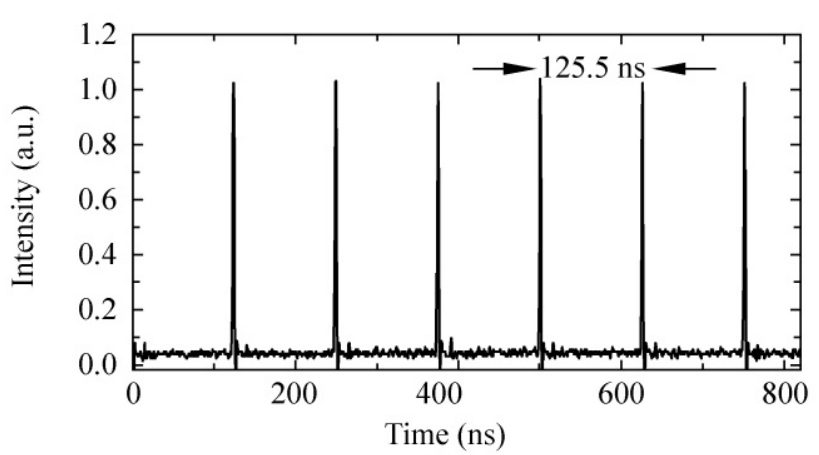

(a)

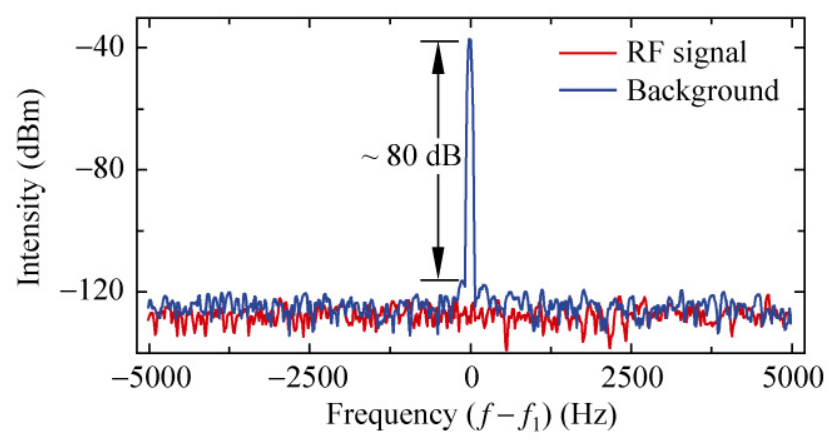

(b)

Figure 6 (a) Output pulse train; (b) RF spectrum, measured around the fundamental repetition rate $f_{1} \sim 8 \mathrm{MHz}$ over $10 \mathrm{kHz}$ with $10 \mathrm{~Hz}$ resolution

\section{Acknowledgements}

We acknowledge funding from a Royal Society Brian Mercer Award for Innovation, the European Research Council (ERC) grant NANOPOTS, Engineering and Physical Sciences Research Council (EPSRC) grants (Nos. EP/GO30480/1 and EP/G042357/1), King's College and Imperial College. ACF is a Royal Society Wolfson Research Merit Award holder.

Open Access: This article is distributed under the terms of the Creative Commons Attribution Noncommercial License which permits any noncommercial use, distribution, and reproduction in any medium, provided the original author(s) and source are credited.

\section{References}

[1] Letokhov, V. S. Laser biology and medicine. Nature 1985, 316, 325-330.

[2] Okhotnikov, O.; Grudinin, A.; Pessa, M. Ultra-fast fibre laser systems based on SESAM technology: New horizons and applications. New J. Phys. 2004, 6, 177. 
[3] Okhotnikov, O. G.; Gomes, L.; Xiang, N.; Jouhti, T.; Grudinin, A. B. Mode-locked ytterbium fiber laser tunable in the 980-1070-nm spectral range. Opt. Lett. 2003, 28, 1522-1524.

[4] Ober, M. H.; Hofer, M.; Hofer, R.; Reider, G. A.; Sucha, G. D.; Fermann, M. E.; Harter, D.; Mendonca, C. A. C.; Chiu, T. H. Widely tunable femtosecond neodymium fiber laser. Opt. Lett. 1995, 20, 2303-2305.

[5] Tamura, K.; Kimura, Y.; Nakazawa, M. Femtosecond pulse generation over $82 \mathrm{~nm}$ wavelength span from passively mode-locked erbium-doped fiber laser. Electron. Lett. 1995, 31, 1062-1063.

[6] Tamura, K.; Doerr, C. R.; Haus, H. A.; Ippen, E. P. Soliton fiber ring laser stablization and tuning with a broad intracavity filter. IEEE Photonics Technol. Lett. 1994, 6, 697-699.

[7] Keller, U. Recent developments in compact ultrafast lasers. Nature 2003, 424, 831-838.

[8] Set, S. Y.; Yaguchi, H.; Tanaka, Y.; Jablonski, M. Ultrafast fiber pulsed lasers incorporating carbon nanotubes. IEEE $J$. Sel. Top. Quantum Electron. 2004, 10, 137-146.

[9] Yamashita, S.; Inoue, Y.; Maruyama, S.; Murakami, Y.; Yaguchi, H.; Jablonski, M.; Set, S. Y. Saturable absorbers incorporating carbon nanotubes directly synthesized onto substrates and fibers and their application to mode-locked fiber lasers. Opt. Lett. 2004, 29, 1581-1583.

[10] Schibli, T. R.; Minoshima, K.; Kataura, H.; Itoga, E.; Minami, N.; Kazaoui, S.; Miyashita, K.; Tokumoto, M.; Sakakibara, Y. Ultrashort pulse-generation by saturable absorber mirrors based on polymer-embedded carbon nanotubes. Opt. Express 2005, 13, 8025-8031.

[11] Nishizawa, N.; Seno, Y.; Sumimura, K.; Sakakibara, Y.; Itoga, E.; Kataura, H.; Itoh, K. All-polarization-maintaining Er-doped ultrashort-pulse fiber laser using carbon nanotube saturable absorber. Opt. Express 2008, 16, 9429-9435.

[12] Rozhin, A. G.; Scardaci, V.; Wang, F.; Hennrich, F.; White, I. H.; Milne, W. I.; Ferrari, A. C. Generation of ultra-fast laser pulses using nanotube mode-lockers. Phys. Stat. Sol. B 2006, 243, 3551-3555.

[13] Shohda, F.; Shirato, T.; Nakazawa, M.; Mata, J.; Tsukamoto, $147 \mathrm{fs}, 51 \mathrm{MHz}$ soliton fiber laser at $1.56 \mu \mathrm{m}$ with a fiberconnector-type SWNT/P3HT saturable absorber. Opt. Express 2008, 16, 20943-20948.

[14] Wang, F.; Rozhin, A. G.; Scardaci, V.; Sun, Z.; Hennrich, F.; White, I. H.; Milne, W. I.; Ferrari, A. C. Widebandtuneable, nanotube mode-locked, fibre laser. Nat. Nanotechnol. 2008, 3, 738-742.

[15] Della Valle, G.; Osellame, R.; Galzerano, G.; Chiodo, N.; Cerullo, G.; Laporta, P.; Svelto, O.; Morgner, U.; Rozhin, A. G.; Scardaci, V.; Ferrari, A. C. Passive mode locking by carbon nanotubes in a femtosecond laser written waveguide laser. Appl. Phys. Lett. 2006, 89, 231115.

[16] Song, Y. W.; Yamashita, S.; Maruyama, S. Single-walled carbon nanotubes for high-energy optical pulse formation. Appl. Phys. Lett. 2008, 92, 021115.

[17] Scardaci, V.; Rozhin, A. G.; Hennrich, F.; Milne, W. I.; Ferrari, A. C. Carbon nanotube-polymer composites for photonic devices. Physica E 2007, 37, 115-118.

[18] Fong, K. H.; Kikuchi, K.; Goh, C. S.; Set, S. Y.; Grange, R.; Haiml, M.; Schlatter, A.; Keller, U. Solid-state Er:Yb:glass laser mode-locked by using single-wall carbon nanotube thin film. Opt. Lett. 2007, 32, 38-40.

[19] Scardaci, V.; Sun, Z.; Wang, F.; Rozhin, A. G.; Hasan, T.; Hennrich, F.; White, I. H.; Milne, W. I.; Ferrari, A. C. Carbon nanotube polycarbonate composites for ultrafast lasers. Adv. Mater. 2008, 20, 4040-4043.

[20] Solodyankin, M. A.; Obraztsova, E. D.; Lobach, A. S.; Chernov, A. I.; Tausenev, A. V.; Konov, V. I.; Dianov, E. M. Mode-locked $1.93 \mu \mathrm{m}$ thulium fiber laser with a carbon nanotube absorber. Opt. Lett. 2008, 33, 1336-1338.

[21] Sun, Z.; Rozhin, A. G.; Wang, F.; Scardaci, V.; Milne, W. I.; White, I. H.; Hennrich, F.; Ferrari, A. C. L-band ultrafast fiber laser mode locked by carbon nanotubes. Appl. Phys. Lett. 2008, 93, 061114.

[22] Martinez, A.; Uchida, S.; Song, Y. W.; Ishigure, T.; Yamashita, S. Fabrication of carbon nanotube-poly-methylmethacrylate composites for nonlinear photonic devices. Opt. Express 2008, 16, 11337-11343.

[23] Sun, Z.; Rozhin, A. G.; Wang, F.; Hasan, T.; Popa, D.; O'Neill, W.; Ferrari, A. C. A compact, high power, ultrafast laser mode-locked by carbon nanotubes. Appl. Phys. Lett. 2009, 95, 253102.

[24] Kurashima, Y.; Yokota, Y.; Miyamoto, I.; Kataura, H.; Sakakibara, Y. Mode-locking nanoporous alumina membrane embedded with carbon nanotube saturable absorber. Appl. Phys. Lett. 2009, 94, 223102.

[25] Uchida, S.; Martinez, A.; Song, Y. W.; Ishigure, T.; Yamashita, S. Carbon nanotube-doped polymer optical fiber. Opt. Lett. 2009, 34, 3077-3079.

[26] Kelleher, E. J. R.; Travers, J. C.; Sun, Z.; Rozhin, A. G.; Ferrari, A. C.; Popov, S. V.; Taylor, J. R. Nanosecondpulse fiber lasers mode-locked with nanotubes. Appl. Phys. Lett. 2009, 95, 111108

[27] Lim, J. K.; Knabe, K.; Tillman, K. A.; Neely, W.; Wang, Y. S.; Amezcua-Correa, R.; Couny, F.; Light, P. S.; Benabid, F.; Knight, J. C.; Corwin, K. L.; Nicholson, J. W.; Washburn, B. R. A phase-stabilized carbon nanotube fiber laser frequency comb. Opt. Express 2009, 17, 14115-14120. 
[28] Kelleher, E. J. R.; Travers, J. C.; Ippen, E. P.; Sun, Z.; Ferrari, A. C.; Popov, S. V.; Taylor, J. R. Generation and direct measurement of giant chirp in a passively mode-locked laser. Opt. Lett. 2009, 34, 3526-3528.

[29] Kieu, K.; Wise, F. W. All-fiber normal-dispersion femtosecond laser. Opt. Express 2008, 16, 11453-11458.

[30] Nicholson, J. W.; Windeler, R. S.; DiGiovanni, D. J. Optically driven deposition of single-walled carbon-nanotube saturable absorbers on optical fiber end-faces. Opt. Express 2007, 15, 9176-9183.

[31] Kelleher, E. J. R.; Travers, J. C.; Sun, Z; Ferrari, A. C.; Golant, K. M.; Popov, S. V.; Taylor, J. R. Bismuth fiber integrated laser mode-locked by carbon nanotubes. Laser Phys. Lett., in press, DOI:10.1002/lapl.201010067.

[32] Hasan, T.; Sun, Z.; Wang, F.; Bonaccorso, F.; Tan, P. H.; Rozhin, A. G.; Ferrari, A. C. Nanotube-polymer composites for ultrafast photonics. Adv. Mater. 2009, 21, 3874-3899.

[33] Sun, Z.; Hasan, T.; Wang, F.; Rozhin, A. G.; White, I. H.; Ferrari, A. C., Ultrafast stretched-pulse fiber laser modelocked by carbon nanotubes. Nano Res. 2010, 3, 404-411.

[34] Martinez, A.; Zhou, K.; Bennion, I.; Yamashita, S. Passive mode-locked lasing by injecting a carbon nanotube-solution in the core of an optical fiber. Opt. Express 2010, 18, 11008-11014.

[35] Cho, W. B.; Yim, J. H.; Choi, S. Y.; Lee, S.; Schmidt, A.; Steinmeyer, G.; Griebner, U.; Petrov, V.; Yeom, D. I.; Kim, K.; Rotermund, F. Boosting the nonlinear optical response of carbon nanotube saturable absorbers for broadband mode-locking of bulk lasers. Adv. Funct. Mater. 2010, 20, 1937-1943.

[36] Senoo, Y.; Nishizawa, N.; Sakakibara, Y.; Sumimura, K.; Itoga, E.; Kataura, H.; Itoh, K. Polarization-maintaining, high-energy, wavelength-tunable, Er-doped ultrashort pulse fiber laser using carbon-nanotube polyimide film. Opt. Express 2009, 17, 20233-20241.

[37] Kivisto, S.; Hakulinen, T.; Kaskela, A.; Aitchison, B.; Brown, D. P.; Nasibulin, A. G.; Kauppinen, E. I.; Harkoen, A.; Okhotnikov, O. G. Carbon nanotube films for ultrafast broadband technology. Opt. Express 2009, 17, 2358-2363.

[38] Sun, Z.; Hasan, T.; Torrisi, F.; Popa, D.; Privitera, G.; Wang, F.; Bonaccorso, F.; Basko, D. M.; Ferrari, A. C. Graphene mode-locked ultratast laser. ACS Nano 2010, 4, 803-810.

[39] Zhang, H.; Tang, D. Y.; Zhao, L. M.; Bao, Q. L.; Loh, K. P. Large energy mode locking of an erbium-doped fiber laser with atomic layer graphene. Opt. Express 2009, 17, 17630-17635.

[40] Bao, Q.; Zhang, H.; Wang, Y.; Ni, Z.; Yan, Y.; Shen, Z. X.; Loh, K. P.; Tang, D. Y. Atomic-layer graphene as a saturable absorber for ultrafast pulsed lasers. Adv. Funct. Mater. 2009,
19, 3077-3083.

[41] Zhang, H.; Bao, Q. L.; Tang, D. Y.; Zhao, L. M.; Loh, K. Large energy soliton erbium-doped fiber laser with a graphenepolymer composite mode locker. Appl. Phys. Lett. 2009, 95, 141103.

[42] Song, Y. W.; Jang, S. Y.; Han, W. S.; Bae, M. K., Graphene mode-lockers for fiber lasers functioned with evanescent field interaction. Appl. Phys. Lett. 2010, 96, 051122.

[43] Tan, W. D.; Su, C. Y.; Knize, R. J.; Xie, G. Q.; Li, L. J.; Tang, D. Y. Mode locking of ceramic Nd: Yttrium aluminum garnet with graphene as a saturable absorber. Appl. Phys. Lett. 2010, 96, 031106.

[44] Bao, Q.; Zhang, H.; Yang, J. X.; Wang, S.; Tang, D. Y.; Jose, R.; Ramakrishna, S.; Lim, C. T.; Loh, K. P. Graphenepolymer nanofiber membrane for ultrafast photonics. $A d v$. Funct. Mater. 2010, 20, 782-791.

[45] Zhang, H.; Tang, D.; Knize, R. J.; Zhao, L.; Bao, Q.; Loh, K. P. Graphene mode locked, wavelength-tunable, dissipative soliton fiber laser. Appl. Phys. Lett. 2010, 96, 111112.

[46] Bonaccorso, F.; Sun, Z.; Hasan, T.; Ferrari, A. C. Graphene photonics and optoelectronics. Nat. Photonics. 2010, 4, 611-622.

[47] Agrawal, G. P. Applications of Nonlinear Fiber Optics.; Academic Press: London, 2001.

[48] Braun, B.; Kärtner, F. X.; Zhang, G.; Moser, M.; Keller, U. 56-ps passively Q-switched diode-pumped microchip laser. Opt. Lett. 1997, 22, 381-383.

[49] Paschotta, R. Encyclopedia of Laser Physics and Technology; Wiley VCH: Weinheim, Germany, 2008.

[50] Small, D. M.; Penkett, S. A.; Chapman, D. Studies on simple and mixed bile salt micelles by nuclear magnetic resonance spectroscopy. Biochim. Biophys. Acta 1969, 176, 178-189.

[51] Sugihara, G.; Shigematsu, D. S.; Nagadome, S.; Lee, S.; Sasaki, Y.; Igimi, H. Thermodynamic study on the Langmuir adsorption of various bile salts including taurine and glycine conjugates onto graphite in water. Langmuir 2000, 16, 1825-1833.

[52] Sasaki, Y.; Igura, T.; Miyassu, Y. I.; Lee, S.; Nagadome, S.; Takiguchi, H.; Sugihara, G. The adsorption behavior of four bile salt species on graphite in water - Evaluation of effective hydrophobicity of bile acids. Colloids Surf. B 1995, 5, 241-247.

[53] Lotya, M.; Hernandez, Y.; King, P. J.; Smith, R. J.; Nicolosi, V.; Karlsson, L. S.; Blighe, F. M.; De, S.; Wang, Z.; McGovern, I. T.; Duesberg, G. S.; Coleman, J. N. Liquid phase production of graphene by exfoliation of graphite in surfactant/water solutions. J. Am. Chem. Soc. 2009, 131, 3611-3620.

[54] De, S.; King, P. J.; Lotya, M.; O’Neill, A.; Doherty, E. M.; 
Hernandez, Y.; Duesberg, G. S.; Coleman, J. N. Flexible, transparent, conducting films of randomly stacked graphene from surfactant-stabilized, oxide-free graphene dispersions. Small 2010, 6, 458-464.

[55] Islam, M. F.; Rojas, E.; Bergey, D. M.; Johnson, A. T.; Yodh, A. G. High weight fraction surfactant solubilization of single-wall carbon nanotubes in water. Nano Lett. 2003, 3, 269-273.

[56] Mak, K. F.; Sfeir, M. Y.; Wu, Y.; Lui, C. H.; Misewich, J. A.; Heinz, T. F. Measurement of the optical conductivity of graphene. Phys. Rev. Lett. 2008, 101, 196405.

[57] Kravets, V. G.; Grigorenko, A. N.; Nair, R. R.; Blake, P.; Anissimova, S.; Novoselov, K. S.; Geim, A. K. Spectroscopic ellipsometry of graphene and an exciton-shifted van Hove peak in absorption. Phys. Rev. B 2010, 81, 155413.

[58] Eberlein, T.; Bangert, U.; Nair, R. R.; Jones, R.; Gass, M.; Bleloch, A. L.; Novoselov, K. S.; Geim, A.; Briddon, P. R. Plasmon spectroscopy of free-standing graphene films. Phys. Rev. B 2008, 77, 233406.

[59] Hasan, T.; Scardaci, V.; Tan, P. H.; Rozhin, A. G.; Milne, W. I.; Ferrari, A. C. Stabilization and "de-bundling" of single-wall carbon nanotube dispersions in N-Methyl-2pyrrolidone (NMP) by polyvinylpyrrolidone (PVP). J. Phys. Chem. C 2007, 111, 12594-12602.

[60] Giordani, S.; Bergin, S. D.; Nicolosi, V.; Lebedkin, S.; Kappes, M. M.; Blau, W. J.; Coleman, J. N. Debundling of single-walled nanotubes by dilution: Observation of large populations of individual nanotubes in amide solvent dispersions. J. Phys. Chem. B 2006, 110, 15708-15718.
[61] Hernandez, Y.; Nicolosi, V.; Lotya, M.; Blighe, F. M.; Sun, Z. Y.; De, S.; McGovern, I. T.; Holland, B.; Byrne, M.; Gun'ko, Y. K.; Boland, J. J.; Niraj, P.; Duesberg, G.; Krishnamurthy, S.; Goodhue, R.; Hutchison, J.; Scardaci, V.; Ferrari, A. C.; Coleman, J. N. High-yield production of graphene by liquid-phase exfoliation of graphite. Nat. Nanotechnol. 2008, 3, 563-568.

[62] Ferrari, A. C.; Meyer, J. C.; Scardaci, V.; Casiraghi, C.; Lazzeri, M.; Mauri, F.; Piscanec, S.; Jiang, D.; Novoselov, K. S.; Roth, S.; Geim, A. K. Raman spectrum of graphene and graphene layers. Phys. Rev. Lett. 2006, 97, 187401.

[63] Ferrari, A. C.; Robertson, J. Interpretation of Raman spectra of disordered and amorphous carbon. Phys. Rev. B 2000, 61, 14095-14107.

[64] Tuinstra, F.; Koenig, J. L. Raman spectrum of graphite. J. Chem. Phys. 1970, 53, 1126-1130.

[65] Casiraghi, C.; Hartschuh, A.; Qian, H.; Piscanec, S.; Georgi, C.; Fasoli, A.; Novoselov, K. S.; Basko, D. M.; Ferrari, A. C. Raman spectroscopy of graphene edges. Nano Lett. 2009, 9 , 1433-1441.

[66] Latil, S.; Meunier, V.; Henrard, L. Massless fermions in multilayer graphitic systems with misoriented layers: $A b$ initio calculations and experimental fingerprints. Phys. Rev. B 2007, 76, 201402.

[67] Dennis, M. L.; Duling, I. N. Experimental study of side band genaration in femtosecond fiber lasers. IEEE J. Quantum Electron. 1994, 30, 1469-1477.

[68] von der Linde, D. Characterization of the noise in continuously operating mode-locked lasers. Appl. Phys. B 1986, 39, 201-217. 